konstanz bei $105^{\circ}$ getrocknet und wieder gewogen (Wägung 3). Die Differenz zwischen beiden Wägungen ( 2 und 3 ), berechnet auf 100 , ist die Hygroskopizität im wasserdampfgesättigten Raum, die zwischen Wägung 1 , und 3 der Wassergehalt im lufttrockenen Zustande. Die Sohleimpapiere werden, wie bereits gesagt, am besten immer zusammen mit den zugehörigen Zellstoffen eingehängt, um etwaige Fehler zu vermeiden, die durch Temperaturunterschiede und damit verbundene Schwankung im Wassergehalt entstehen könnten.

Durch längeres Erhitzen auf $120^{\circ}$ läßt sich die Fähigkeit schleimiger Stoffe, in erhöhtem Maße Wasser aufzunehmen, bedeutend herabsetzen. Die kolloidartigen Cellulosedextrine werden dann

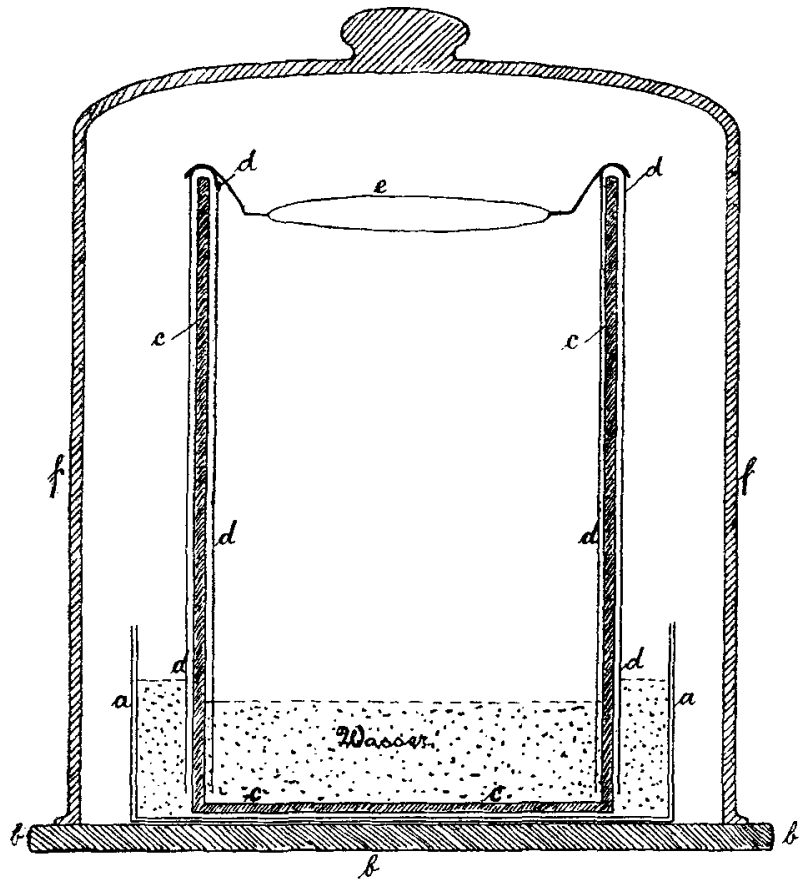

auseheinend irreversibel. Um genauere Zahhlen für diese Vorgänge zu gewinnen, wurden die Papierstreifen nach der Wägung 3 2 Stunden bei $120^{\circ}$ erhitzt, sodann wieder 4 Stunden in dem beschriebenen Apparat im wasserdampfgesättigten Raum anfbewahrt und schließlich wieder gewogen (Wägung 4). Die Differenz zwischen Wägung 4 und Wägung 3 ist die Hygroskopizität in wasserdampfgesättigter Luft, $\mathbf{n}$ a $\mathbf{~ h ~ d e m ~ T r o c k n e n ~ b e i ~} 120^{\circ}$. Die dafür gefundenen Zahlen sind ebenfalls in der Tafel XI niedergelegt.

Auch die vollkommenen Schleime, die aus nach D. R. P. Nr.303498 zermürbten Zellstoffen hergestellt waren, wurden 2 Stunden bei $120^{\circ}$ getrocknet und dann nach der ersten Versuchsanordnung bis zur Gewichtskonstanz einige Wochen der wasserdampfgesättigten Luft ansgesetzt. Der dann ermittelte Wassergehalt ist in Tafel XII in der Rubrik a angegeben.

Werden die schleimbildenden Stoffe durch längeres Dämpfen mit heißem Wasser ganz oder wenigstens zum größten Teil entfernt, was aus der Abnahme des Reduktionsvermögens hervorzugehen scheint, so geht die Aufnahmefähigkeit für Wasser ebenfalls stark zurück, wofür die Zahlen in Tafel XII in der Rubrik b zu finden sind. Ähnlich, wenn auch nicht so stark, wirkt lange fortgesetztes Auswaschen mit kaltem Wasser, das die schleimbildenden, dextrinartigen Körper ebenfalls zum Teil entfernt (Tafel XII c).

Auffallend ist der Widerspruch zwischen den hier genannten Befunden für die hohe Wasseraufnahmefähigkeit der zermürbten (e. Tafel X, b und d) Zellstoffe, die doch nichts anderes sind, als Hydrocellulosen, und der geringen Hygroskopizität der Hydrocellulose aus Baumwolle nach $G$ ir a r d in wasserdampfgesättigter Iuft. Eine nach G ir a $\mathrm{rd}$ hergestellte Hydrocellulose hat nach unserer Bestimmung eine Hygroskopizität im wasserdanjpfgesättigten Raum von $11 \%$. Q vis t ${ }^{1}$ ) hat eine Reihe Hydrocellulosen, die nach Girard aus Sulfitzellstoffen hergestellt waren, untersucht und Werte von 9,2 bis $11,9 \%$ erhalten. Allerdings ist dabei zu bemerken, daß die nach D. R. P. Nr. 303498 zermürbten Zellstoffe nicht ausgewaschen sind, dagegen $Q$ v i s t zuerst mit Wasser und dann mit verdünnter Sodalösung auswäscht. Er hat deshalb auch nur Kupferzahlen von $4,3-5,1$ gefundien $^{2}$ )

Das Hauptergebnis der. Untersuchungen nach physikalischer Richtung hin ist, daß der Stoff durch anhaltende $\mathrm{me} \mathrm{ch}$ a $\mathrm{n}$ i s $\mathrm{chc}$ Bearbeitung die Fähigkeit erhält, Wa s s e $r$ in $g$ ro $B$ e r Me n ge in wasserdampfgesättigter Luft a uf $\mathrm{z}$ u $n \bullet \mathrm{hm} \bullet \mathrm{n}$, und daB durch Trocknen bei $120^{\circ}$ diese Fähigkeit a u f $g$ e h o b e n wird, indem die kolloidartigen Bestandteile des Schleimes ir.r $\theta$ v ers i b el werden.

Zum Schluß muß betont werden, da $B$ vorstehende Untersuchungen sich auf ungebleichte Zellstoffe beziehen. Bei den gebleichten Zellstoffen scheinen die Verhältnisse noch etwas verwickelter zu liegen, da die Wirkung der Bleiche hinzukommt. Untersuchungen über Schleime aus oxydierten Zellstoffen sind in Aussicht genommen.

Bei den drei Untersuchungen: ,Zur Kenntnis der Zellstoff schleime," wurden wir bei der Ausführung der Analysen in dankens. werter Weise von den Damen Ba ermann und Bornefeld im Sommer, Rich ter und $S$ ch o e n w a l dim Winter, unterstutzt.

[A. 2066 .]

\section{Beiträge zur Gewichtsanalyse $\mathrm{X} \mathbf{I}^{\mathrm{1}}$ ).}

XV. Bestimmung der Schwefelsäure. (Zweite Abhandlung.)

Von L. W. WiNkLER, Budapest. (kingeg. 20./11. 1919.)

Die Untersuchungen, die Bestimmung der Schwefelsäure al Bariumsulfat ${ }^{2}$ ) betreffend, wurden fortgesetzt und unter anderem nochmals geprifft, wie sich das Ergebnis mit dem Säuregrad der zn fällenden Lösung ändert. Die Analysen wurden unter Leitung des Verfassers durch Herrn Cand. phil. E. \$ $\mathrm{ch} \mathrm{u} \mathrm{le} \mathrm{k} \mathrm{ausgeführt}$

Bei den vorliegenden Untersuchungen diente als Ausgangslösung annähernd $1 / 10-\mathrm{n} . \mathrm{S} \mathrm{ch}$ wef els $\mathrm{äure} \mathrm{e}^{3}$, deren Stärke durch Einstellen auf reinstes trockenes Kaliumbicarbonat ${ }^{4}$ ) zu 0,09897 normal gefunden wurde. Von dieser Lösung gelangten Anteile von $25 \mathrm{ccm}$ zur Abmessung, die mit $\mathrm{KHCO}_{3}$, mit $\mathrm{NH}_{3}$ oder mit $\mathrm{Na}_{2} \mathrm{CO}_{3}$ genau gesättigt wurden (Endanzeiger Methylorange). Die Salzlösungen wurden teils ohne, teils nach dem Zusatz gemessener Mengen n.-Salzsäure auf $100 \mathrm{ccm}$ verdünnt. Das Fällen wurde mit $5 \mathrm{ccm} ; 10 \%$ iger" Bariumchloridlösung $\left(10,0 \mathrm{~g} \mathrm{BaCl}, 2 \mathrm{H}_{2} \mathrm{O}\right.$ gelöst auf $100 \mathrm{~cm}$ ) vorgenommen, indem das Fällungsmittel tropfenweise zu der in ruhigem Kochen befindlichen Untersuchungslösung hinzugefügt wurde; nach dem Fällen wurde die Flüssigkeit noch $5^{\prime}$ lang im Sieden erhalten. Das Sammeln des Niederschlages in ,Kelchtrichter" auf einem Wattebausch erfolgte nach 24 Stunden,

1) Q v is t, Papierfabrikant 17, 818 [1919].

2) Bei den Hydrocellulosen ist für gewöhnliche Luftfeuchtigkeit häufig ein Wert gefunden worden, der untcr dem für Baumwoll liegt. Die Hygroskopizitätsbestimmungen bei gewöhnlicher Luftfeuchtigkeit sind aber wenig zuverlässig, weil genaue Luftfeuchtigkeitsmessungen nicht ausgeführt werden. Eine Erörtorung der pinschlägigen Verhältnisse würde hier zu weit führen.

1) Vgl. Angew. Chem. 30, I, 251 und 301 [1917]; 31, 1, 46, 80, $101,187,211$ und 214 [1918]; 32, I, 24, 99 und 122 [1919]

2) Angew. Chem. 30, I, 251 und 259 [1917].

3) Aus konz. Schwefelsäure bereitet, die durch Úberdampfen gereinigt wurde.

4) Vgl. Angew. Chem. 28, I, 264 [1915]. - Bei dieser Gelegenheit möge erwähnt werden, daß man das Kaliumbicarbonat besser, nicht wie früher angegeben, über Caloiumchlorid sondern über geglühtem Glaubersalz in den mit Kohlendioxyd gefüllten Trockengefäß (Exsiccator) trocknet, da bei längerem Sttehen iibe Calciumchlorid in das Salz Spuren von Chlor gelangen.

Zahlentafel XII.

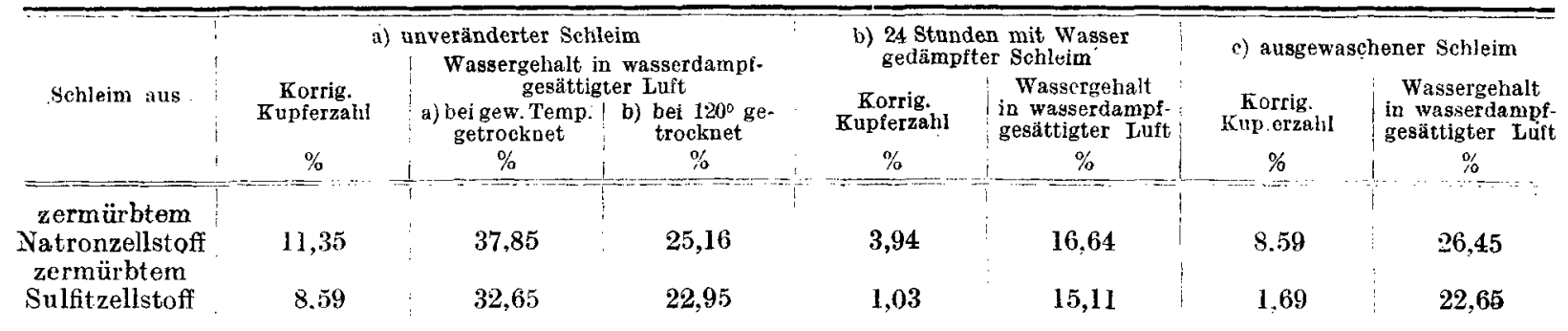


zum Auswaschen des Niederschlages wurden $25 \mathrm{ccm}$ kaltes und ebensoviel heiBes Wasser benutzt.

Es wurde sowohl die Menge dés bei $130^{\circ}$ getrockneten, als auch die des geglühten Niederschlags bestimmt. Die auf Grundlage der maßanalytischen Bestimmung berechnete Menge $\mathrm{BaSO}_{4}$ beträgt 288,8 mg. Die Ergebnisse enthalten folgende Zahlenreihen; die einzelnen Zahlen sind Mittelwerte aus 3-6 Bestimmungen:

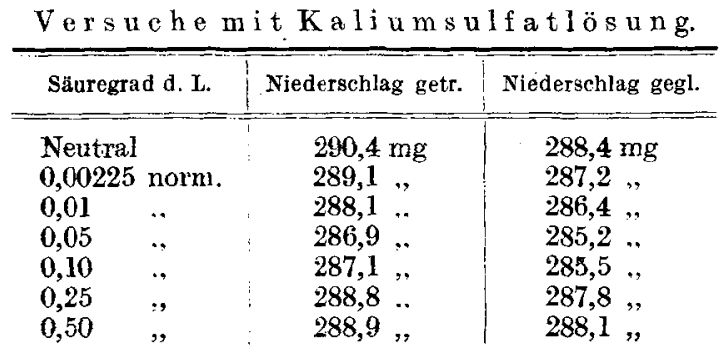

Die Verhältnisse lassen sich besser überblicken, wenn man die berechnete Menge $\mathrm{BaSO}_{4}=100$ setzt; die Zahlen der letzten Zeile sind aus den Versuchsergebnissen der ersten Abhandlung berechnet:

\begin{tabular}{|c|c|c|c|c|}
\hline \multicolumn{2}{|c|}{ Säıregrad d. $L$. } & Niederschlag getr. & Niedersctilag gegl. & Githverlust \\
\hline \multicolumn{2}{|c|}{ Neutral } & 100,56 & 99,86 & 0,70 \\
\hline \multicolumn{2}{|c|}{0,00225 norm. } & 100,10 & 99,44 & 0,66 \\
\hline 0,01 &. & 99,76 & 99,17 & 0,59 \\
\hline 0,05 &. & 99,34 & 98,75 & 0,59 \\
\hline 0,10 & . & 99,41 & 98,86 & 0,55 \\
\hline 0,25 & 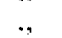 & 100,00 & 99,66 & 0,34 \\
\hline 0,50 & , & 100,03 & 99,76 & 0,27 \\
\hline 1,00 &, & 99,10 & 98,80 & 0,30 \\
\hline
\end{tabular}

Die durch Zunahme des Säuregrades bedingte Gewichtsänderung der Niederschläge zwischen den Grenzen 0,0 und 1,0 norm. entspricht also dem Verlauf einer We ll e n li ni e. Der Glühverlust der getrockneten Niederschläge dagegen ist um so geringer, aus je saurer Lösung der Niederschlag gefällt wurde.

Wurden die $25 \mathrm{ccm}$ betragenden Anteile der Schwefelsäure mit A m m on iak gesättigt, und die Versuche genau so, wie die mit Kaliumsulfatlösung ausgeführt, so war das Ergebnis:

\begin{tabular}{|c|c|c|}
\hline Säuregrad d. L. & Niederschlag getr. & Niederschlag gegl. \\
\hline Neutral & $290,5 \mathrm{mg}$ & $287,7 \mathrm{mg}$ \\
\hline 0,00225 norm. & $290,2 \ldots$ & $287,5,$. \\
\hline 0,01 & $288,2 \ldots$ & 286,5, \\
\hline 0,05 & 287,3, & 286,0, \\
\hline
\end{tabular}

Fs zeigt sich also, daß es fast auf eins herauskommt, ob manmit KaIium-oder Ammoniumsulfatlö. s u $\mathrm{ng}$ arbeitet.

Wurden dagegen die Schwefelsäureproben mit $N$ atrium e a r b o n a t gesättigt, so konnten geringe Un e g e $1 \mathrm{~m}$ ä $B$ i g $k$ e it en beobachtet werden, wie folgende Zahlen zeigen:

\begin{tabular}{|c|c|c|}
\hline Säuregrad d. L. & Niederschlag getr. & Niederschlag gegl. \\
\hline Neutral & $291,5 \mathrm{mg}$ & $289,4 \mathrm{mg}$ \\
\hline 0,00225 norm. & 288,9, & 287,1, \\
\hline $0,01 \quad$, & 289,5 & 288,1, \\
\hline 0,05 & 288,4 & 287,5, \\
\hline
\end{tabular}

Wird beim Fällen die Bariumehloridlösung i m großen Überseh u B $(50 \mathrm{ccm})$ genommen, so vergröhert sich kaum das Ergebnis (etwa um $1 \mathrm{mg}$ ), wenn man das Fällungsmittel tropfenweise zur kochendheißen Lösung hinzufügt. Natürlich ändern sich die Verhältnisse westntlich, wenn man die Bariumchloridlösung in großem Überschuß in einem Guß zur Lösung gibt, wobei ansehnliche Mengen Bariumchlorid mitgerissen werclen.

Wartet man nicht bis zum anderen Tage, sondern nimmt das Seinen schon nach 2 Stunden vor, so ist das Gewicht des Niederschlags uin ein geringes kleiner (ctwa $0,5 \mathrm{mg}$ ), besonders wenn die Lösurg stärker sautr war; auch schwanken die Werte der Einzelbestimmungen etwas mehr.

Es wurden auch einige Vorsuche unternomme'l, um zu erfahren, wic aroBdie Menge der schwefels äure ist, die beim Glühen aus dem aus schwach saurer Lö sung gefällten undgetrockneten Niedersehlag entweicht. Der Niederschlag selbst wurde aus kochendheißè Kalium-, Ammonium- oder Natriumsulfatlösung gefällt, wclch Lösungen durch Salzsäurezusatz auf den Säuregrad 0,05 n. gebracht wurden. - Die Versuchsanordnung zeigt nachstehende Zeichnung:

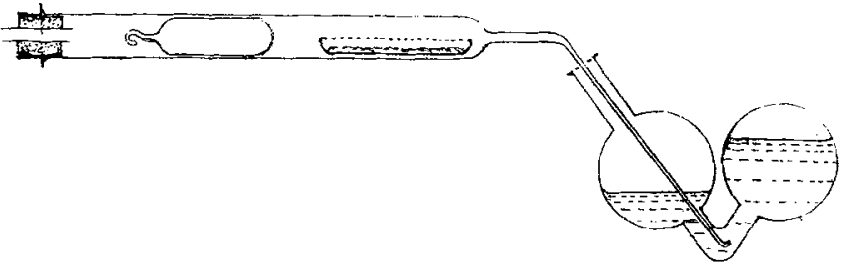

Die gut ausgewaschene und vorher bei $130^{\circ}$ getrocknete Nieder schlagsprobe von etwa $1 \mathrm{~g}$ befand sich in einem Platinsehiffchen; die etwa $200 \mathrm{ccm}$ fassende Auffangvorrichtung war mit reinstem de stilliertem Wasser beschickt. Durch die Kaliröhre wurde in lang samem Strome trockene Luft geleitet und gleichzeitig der vorder Teil der Röhre, wo sich das Schiffchen befand, anfangs gelinde, dann bis zur Rotglut erhitzt. Es konnte mit dem Auge das Entweichen von Schwefelsäuredämpfen beobachtet werden, nämlich der bauchige Teil der Auffangvorrichtung fülite sich mit einem schweren Nebel, der durch das Wasser nur allmählich gelöst wurde. Um die in dem verjüngten Teil der Kaliröhre allenfalls niedergeschlagene Schwefelsäure nicht zu verlieren, wurde nach Beenden des Versuches das Wasser bis zu dem wagerechten Teil des engen Rohres aufgesangt, dann wieder ausgetrieben.

Das in der Auffangvorrichtung befindliche, sauer gewordene Wasser wurde in einer Platinschale eingetrocknet, der Ruckstand in $20 \mathrm{ccm}$ Wasser gelöst und die Schwefelsäure mit $1 \mathrm{ccm}$ Bariumchloridlösung heiß gefällt. Am anderen Tage wurde der Niecterschlag in einem ganz kleinen "Kelchtrichter" auf Watte gesammelt, mit $20 \mathrm{ccm}$ Wasser ausgewaschen, getrocknet und gewogen. Die der Reihe nach aus der Kalium-, Ammonium- und Natriumsulfatlösung erhaltenen und zu den Versuchen benutzten Niederschlag. proben sind mit $a$, die aus dem schwefelsäurehaltigen Wasser gewonnenen Bariumsulfatmengen mit $b$ bezeichnet:

\begin{tabular}{cl}
$a$ & \multicolumn{1}{c}{$b$} \\
$1,1342 \mathrm{~g}$ & $5,4 \mathrm{mg}$ \\
1,2312, & 3,8, \\
1,1348, & 3,2, ?
\end{tabular}

Die auf maßanalytischem und gewichtsanalytischem Wege erhaltenen, mit verschiedenartigen Versuchsfehlern behafteten Werte sind eigentlich unvergleichbar: es dürfen streng genommen die mit Kalium-, Ammonium- und Natriumsulfatlösungen erhaltenen Werte nur untereinander verglichen werden. Eben deshalb wurden, um die in der ersten Abhandlung erhaltenen Ergebnisse zu überprïfen, wicder einige Versuche mit einer solchen Kaliumsulfatlösung ausgeführt, die aus allerreinstem, sehrseharf getrocknetem $\mathrm{Kalinms}$ u $\mathrm{fat}^{5}$ ) bereitet wurde. Von dem Salze wurden 4,4559 $\mathrm{g}$ in Wasser auf $1000 \mathrm{ccm}$ gelöst und von dieser Lösung Antcile von $50 \mathrm{ccm}$ zu den Versuchen genonmen, dje auf $100 \mathrm{ccm}$ verdünnt und mit $1,0 \mathrm{ccm}$ norm. Salzsäure angesäuert wurden; die berechnete Menge $\mathrm{BaSO}_{4}$ beträgt $298,45 \mathrm{mg}$. Im übrigen wurde so verfahren, wie bei den weiter oben beschriebenen Versuchen, mit dem Unterschiede, daß das Trocknen des Niederschlages anfänglich bei $100^{\circ}$, später bei $130^{\circ}$ vorgenommen wurde. Das Gewicht der Niederschlagsproben betrug der Reibe nach im Mittel aus je 6 Versuchen 297,10 und 297,10 , geglüht $295,50 \mathrm{mg}$.

Es zeigt sich also, daß das Ergebnis dasselbe ist, wenn man das Trocknen bei 100 oder bei $130^{\circ}$ vornimmt. Der höhere Wärmegrad ist vorteilhafter. Trocknet man nämlich bei $100^{\circ}$, so sind $3-4$ Stunden nötig, trocknet man bei $130^{\circ}$, so genügen 2 Stunden bis zum unveränderlichen Gewichte. Aus den Versuchen ergibt sich ferner, daB, wenn man mit einer Kaliumsulfatlösung von dem Säuregrade 0,01 norm. arbeitet, man den etwa $0,3 \mathrm{~g}$ schweren getrockneten Niederschlag mit 1,0045, den geglühten mit 1,0099 zu multiplizieren hat, damit das Ergebnis richtig sei ; früher wurden die Zahlen 1,0046 und 1,0086 gefunden. - Die Versuche werden fortgesetzt. [A. 187.]

5) Um zu einem wirklich wasserfreien Salze zu gelangen, genügt es durchaus nicht, das Kaliumsulfat einfach im Trockenschrank zu trocknen. Man muß vielmehr das Salz im Achatmörser zu feinstem Pulver zerreiben und dieses in einer Platinschale unter fortwährendem Umrühren über der Gasflamme so lange erbitzen, bis es nicht mehr knistert, und der Boden der Schale eben zu glühen beginnt. 